\title{
Reinforcement alone does not explain increased reproductive isolation in sympatry
}

Daniel R. Matute* and Brandon S. Cooper ${ }^{\dagger}$

*Biology Department

University of North Carolina

250 Bell Tower Drive, Genome Sciences Building

Chapel Hill, NC 27510

${ }^{\dagger}$ Division of Biological Sciences

University of Montana

32 Campus Dr.

Missoula, MT 59812

Email: dmatute@email.unc.edu, brandon.cooper@umontana.edu

\section{Classification}

Biological Sciences. Evolution

\section{Keywords}

adaptation, Bufo, Drosophila, Lepidoptera, natural selection, speciation

\section{Author Contributions}

DRM designed the research, analyzed and interpreted the data, wrote the paper, and coordinated the study. BSC designed the research, contributed to data analysis and interpretation, and wrote the paper.

This PDF file includes:

Main Text

Figures 1 to 3 


\section{Abstract}

3 Comparative studies of reproductive isolation $(\mathrm{RI})$ by Coyne and Orr $(1,2)$ and others

4 indicate a significant role for reinforcing natural selection in Drosophila speciation. The

5 reinforcement hypothesis predicts increased prezygotic, but not postzygotic, RI between

6 sympatric species pairs in response to maladaptive hybridization. We revisit this

7 hypothesis and others using additional Drosophila, Lepidopteran, and toad (Bufo) data.

8 In contrast to the predictions of reinforcement, we find increased premating and

9 postzygotic RI between sympatric Drosophila species, including between recently

10 diverged species pairs, as defined by Coyne and Orr (1) and others (i.e., $\left.D_{\text {Nei }}<0.5\right)$.

11 However, at slightly lower divergence thresholds increased postzygotic RI in sympatry is

12 not statistically significant, while increased premating $\mathrm{Rl}$ is, generally in agreement with

13 the predictions of reinforcement. While premating data are unavailable, postzygotic $\mathrm{RI}$ is

14 also increased between sympatric Lepidopteran and toad (Bufo) species. We find only

15 modest support for "concordant asymmetries" in premating and postzygotic RI between

16 sympatric Drosophila, described by others as uniquely supporting reinforcement.

17 Finally, the proportion of geographic range overlap shared by species is positively

18 associated with the magnitude of premating $\mathrm{RI}$ as predicted by reinforcement, but it is also positively associated with postzygotic RI, which cannot be explained by reinforcement. Taken together, our results demonstrate that comparisons of premating

21 and postzygotic $\mathrm{RI}$ in sympatry depend greatly on divergence time, and suggest that

22 fusion, extinction, and/or other mechanisms must combine with reinforcement to 23 generate these patterns. 


\section{Significance Statement}

34 Understanding mechanisms underlying the origin of species remains a central goal of

35 biology. By combining laboratory estimates of premating and postzygotic reproductive

36 isolation ( $\mathrm{Rl}$ ) with phylogenetic hypotheses, geographic range data, and genetic

37 divergence estimates, the fundamental meta-analyses of Coyne and Orr provided

38 compelling support that reinforcement contributes significantly to Drosophila speciation.

39 Using additional Drosophila data, we demonstrate increased premating and postzygotic

$40 \mathrm{Rl}$ in sympatry. Postzygotic $\mathrm{RI}$ is also increased in sympatry between Lepidopteran and

41 toad species. We also find only modest support for patterns previously described as

42 uniquely supporting reinforcement. Together, our analyses demonstrate that

43 comparisons of premating and postzygotic RI depend on divergence time, and suggest

44 that fusion, extinction, and/or other mechanisms combine with reinforcement to produce

45 these patterns. 


\section{Introduction}

Speciation, the process in which one species splits into two, results from the accumulation of genetic differences and the evolution of reproductive isolation (RI) (3, 4). In broad terms, barriers to gene flow can be classified based on when they occur in the reproductive cycle, commonly in relation to the generation of a fertilized zygote $(5$, 6). These barriers fall into two general categories: prezygotic and postzygotic RI. Prezygotic $\mathrm{RI}$ includes all ecological, behavioral, mechanical, and gametic incompatibilities that occur before a zygote is formed (5), with most focus placed on premating traits (e.g., mate discrimination). Postzygotic barriers occur after a hybrid zygote is formed and include phenotypes as extreme as hybrid sterility and inviability (7, 8), but also more nuanced traits, such as hybrid behavioral defects (9-11) or delays in development (12-14). Coyne and Orr [(1, 2); hereafter "C\&O"] completed the first indepth meta-analyses of speciation that combined estimates of genetic divergence, premating RI, and hybrid viability and fertility in Drosophila with phylogenetic and geographic information. Their work demonstrated that both premating and postzygotic $\mathrm{RI}$ become stronger as the genetic distance between species increases, and that premating $\mathrm{Rl}$ accumulates faster in sympatry.

Comparative analyses of speciation have greatly influenced our understanding of speciation (15). Analyses similar to those of $C \& O$, and in some cases similar datasets, have revealed seminal aspects of how speciation proceeds. Studies of the rates of evolution of premating and postmating RI between Drosophila species pairs have enabled us to understand the role of sex chromosomes in speciation (16-18), and the potential drivers of speciation (19-22). Indeed, C\&O sparked a cottage industry to study $\mathrm{RI}$ in different groups which has demonstrated that the monotonic increase in $\mathrm{RI}$ as divergence accrues is not specific to Drosophila, occurring in virtually all studied taxa $(23,24)$.

Arguably the most influential finding of $\mathrm{C} \& \mathrm{O}$ was that in contrast to postzygotic $\mathrm{RI}$, mate discrimination is more pronounced between sympatric than between allopatric species pairs of the same age. These results were taken as evidence that natural selection favors the evolution of premating $\mathrm{RI}$ when allopatric species come into secondary contact. In this process, coined reinforcement, premating $\mathrm{Rl}$ is strengthened 
as a byproduct of natural selection against hybridization (25-27). These analyses and others led to the conclusion that reinforcement is common during speciation $(16,28)$. As C\&O $(1,2)$ noted, "any factor that reduces gene flow should inhibit fusion or extinction". Observing increased prezygotic and postzygotic RI in sympatry, particularly for recently diverged species, would support a fusion and/or extinction hypothesis since only populations separated by strong mating discrimination may persist $(1,29,30)$.

Additional premating and postzygotic RI estimates from Drosophila, in combination with estimates of postzygotic RI between Lepidopteran and between toad (Bufo) species, provide the opportunity to revisit and test the predictions of reinforcement and related hypotheses. First, and in contrast to $\mathrm{C} \& \mathrm{O}$, we find increased premating and postzygotic $\mathrm{RI}$ between recently diverged sympatric relative to allopatric Drosophila pairs using their divergence threshold $\left(D_{\mathrm{Nei}}<0.5\right)$; but at slightly lower thresholds increased postzygotic $\mathrm{Rl}$ in sympatry is not statistically significant, while increased premating $\mathrm{Rl}$ is, in agreement with the predictions of reinforcement. Second, while butterfly and toad data do not include estimates of premating Rl, we also observe increased postzygotic $\mathrm{Rl}$ in sympatry for these taxa, including for the most recently diverged toads. Third, our expanded Drosophila analyses find only modest evidence for stronger premating $\mathrm{RI}$ among the more costly reciprocal matings. Fourth, premating $\mathrm{RI}$ is positively associated with the proportion of geographic range overlap, but so is postzygotic RI, which cannot be explained by reinforcement. Our results suggest that fusion, extinction, and/or other mechanisms combine with reinforcement to generate the patterns we observe.

\section{Results}

\section{Increased premating and postzygotic RI in sympatry}

$\mathrm{RI}$ increases as species diverge, and several efforts have reported a negative correlation between parental species divergence and both the strength of mating discrimination (1, 2, 31) and the fitness of resulting hybrids (e.g., (32-34), reviewed in $(15,23,24,35))$. We fit three types of models to study the evolution of RI between diverging species. For both premating and postzygotic RI in Drosophila, four-parameter 
111 logistic (henceforth abbreviated 4PL) models fit the data better than do linear or logistic 112 models, for both allopatric and sympatric species pairs (Table S1). The 4PL model was 113 also the best fit for postzygotic RI in toads and Lepidopterans, for both allopatric and 114 sympatric species pairs. The 4PL model meets the biological expectation of a waiting 115 time for mutations that generate either hybrid incompatibility (36-38) or behavioral isolation (31), followed by a rapid accumulation of $\mathrm{RI}$ that eventually asymptotes. Please note that even though Nei's $D$, our metric of genetic differentiation between Drosophila

118 parental species, is not a perfect predictor of the extent of differentiation, it represents a good proxy (Figure S1 in (9)).

Next, we re-assessed support for the hypothesis that reinforcement has driven the evolution of premating $\mathrm{RI}$ in sympatry for Drosophila. We leveraged the information provided by our model fits and compared the values of the lower asymptote, the upper asymptote, and the inflection point for sympatric and allopatric species pairs, for both premating and postzygotic RI (Figure 1A and B, respectively). Table S2 shows pairwise comparisons for the regression parameters for allopatric and sympatric species. To determine whether isolation evolved faster in sympatric species, we compared $c$, the inflection point of the 4PL model, and a proxy for how fast RI completes, for regressions using either sympatric or allopatric species. Consistent with previous studies $(1,2)$ premating $\mathrm{R}$ I between sympatric Drosophila species pairs reaches $c$ earlier than allopatric species pairs ( $c_{\text {Sympatric-Drosophila-premating }}=0.059$; $c_{\text {Allopatric-Drosophila-premating }}=0.318$; Wilcoxon rank sum test with continuity correction: $W=978,080, P<1 \times 10^{-10}$; Figure $1 \mathrm{C}$ ). The rate of increase once the inflection point is reached (Hill's slope, or $b$ ) is higher for allopatric pairs which eventually leads to similar $d$ (asymptote) values. In Figure S1, we present the other regression parameters: $a, b$, and $d$. Surprisingly, we observed that for postzygotic RI, $c$ is lower and $b$ is higher for sympatric than for allopatric pairs,

136 indicating that postzygotic $\mathrm{Rl}$ also increases faster as divergence accrues between

137 sympatric species ( $C_{\text {Sympatric-Drosophila-postzygotic }}=0.439 ; C_{\text {Allopatric-Drosophila- postzygotic }}=0.751$;

138 Wilcoxon rank sum test with continuity correction: $W=993,050, P<1 \times 10^{-10}$; Figure 139 1D). These results suggest that both premating and postzygotic RI increase faster 140 between sympatric than between allopatric species pairs. We find similar results for 141 both premating and postzygotic RI in Drosophila after using stringent subsamplings at 
142 the species-group level (Premating: $c_{\text {Sympatric-Drosophila }}=0.141 ; c_{\text {Allopatric- Drosophila }}=0.394, \mathrm{~W}$

$143=16,917 ; P=3.654 \times 10^{-11} ;$ Postzygotic: $C_{\text {Sympatric-Drosophila }}=0.38 ; C_{\text {Allopatric- } \text { Drosophila }}=$

$144 \quad 0.610, \mathrm{~W}=8,342 ; P=3.613 \times 10^{-10}$; Figure S2).

$145 \quad$ Next, we fit a phylogenetically informed linear regression. The models included

146 an effect for geographic origin and an interaction between origin and genetic distance. If

$147 \mathrm{RI}$ evolves similarly in sympatry and allopatry, as expected for postzygotic RI under a

148 scenario of pure-reinforcement driving the evolution of strengthened RI in sympatry,

149 then effects of both origin and the interaction should be negligible. For premating RI, we

150 found that sympatric species display generally stronger premating $\mathrm{RI}$ than do allopatric

151 species $(95 \% \mathrm{Cl}=[0.245,0.464], P<0.001)$, but the increase of premating $\mathrm{Rl}$ is slower

152 in sympatric species $(95 \% \mathrm{Cl}=[-0.323,0.058], P=0.014$; Table S3). For postzygotic

$153 \mathrm{RI}$, we found that sympatric and allopatric species have a similar level of postzygotic RI

$154(95 \% \mathrm{Cl}=[-0.121,0.198], P=0.660)$, but that postzygotic $\mathrm{RI}$ increases faster with

155 genetic distance in sympatry $(95 \% \mathrm{Cl}=[0.138,0.699], P=0.002 ;$ Table S3). These

156 results are consistent with previous reports of stronger $\mathrm{Rl}$ in sympatry using a linear

157 model of accumulation of premating and postzygotic RI (39). They are also consistent

158 with the results from the uncorrected datasets, demonstrating that in Drosophila

159 premating and postzygotic RI both evolve faster in sympatry.

While premating data are not available, we also studied these same parameters for postzygotic RI between Lepidopteran species and Bufo toads (Figure 2). The 4PL 162 regression results for these taxa are similar to those in Drosophila (Figure 2A and B).

163 The inflection point is lower for sympatric than for allopatric species pairs for both

164 Lepidopterans ( $C_{\text {Sympatric-Lepidopterans }}=0.686$; $C_{\text {Allopatric- Lepidopterans }}=0.834, W=89,102, P<$

$1651 \times 10^{-10}$; Figure 2C) and Bufo toads (C Sympatric-Toads $=0.028 ; c_{\text {Allopatric-Toads }}=0.038, W=$

$166130,280, P<1 \times 10^{-10}$; Figure 2D). For Lepidopterans, $b$ is much higher for sympatric

167 than for allopatric species pairs $\left(b_{\text {Sympatric-Lepidopterans }}=242.1179 ; b_{\text {Allopatric-Lepidopterans }}=\right.$

$1687.321, W=130,280, P<1 \times 10^{-10}$; Figure S3), which reflects that substantial

169 postzygotic $\mathrm{Rl}$ is rare between allopatric species pairs even in the most divergent

170 crosses (i.e., Papilio xuthus $\times$ P. glaucus being an exception, Nei's $D=1.161$ ) .

171 Subsampling by genus produces a similar result. While $c$ in sympatric species is similar

172 to the complete dataset $\left(c_{\text {Sympatric-Lepidopterans }}=0.887\right), b$ is lower but positive $\left(b_{\text {Sympatric- }}\right.$ 
173 Lepidopterans $=5.801)$. Neither of these two parameters could be calculated for allopatric

174 species because there was no increase in the magnitude of postzygotic RI over genetic

175 distance for these species, and the 4PL regressions did not converge.

176 For Bufo, $b$ is higher for allopatric than for sympatric species pairs ( $b_{\text {Sympatric-Toads }}$

$177=1.734 ; b_{\text {Allopatric-Toads }}=2.899, W=26,916, P<1 \times 10^{-10}$; Figure S3), which is consistent

178 with the earlier inflection point for sympatric pairs and the similar asymptote of both

179 cases ( $d=1$, Figure S3). A subsampling by subgenus was not possible for Bufo (See

180 Methods).

181 We followed up the analyses in these two groups with a phylogenetically

182 corrected linear regression. In the case of Lepidopterans, sympatric species have

183 slightly higher postzygotic RI compared with allopatric species $(95 \% \mathrm{Cl}=[-0.018$,

1840.257 ], $P=0.076$ ), similar to premating $\mathrm{Rl}$ in Drosophila. The increase of inviability with

185 genetic distance is faster for sympatric species than for allopatric species $(95 \% \mathrm{Cl}=[-$

$1860.674,-0.094], P=0.014$; Table S4). In Bufo, we find that postzygotic $\mathrm{RI}$ is generally

187 lower in sympatric species $(95 \% \mathrm{Cl}=[-0.272,-0.032], P=0.006)$, but that $\mathrm{RI}$ increases

188 faster with genetic distance between sympatric species pairs $(95 \% \mathrm{Cl}=[0.486,3.637]$,

$189 P=0.012 ;$ Table S4). These regressions are more limited than 4PL regressions, as

190 linear regressions do not differentiate between the inflection point $(c)$ and the rate of

191 increase with genetic distance (b). Nonetheless, these results are consistent with the

192 4PL results in that the rate of evolution of postzygotic $\mathrm{Rl}$ is not equivalent in allopatric

193 and sympatric species.

194 In sum, our results indicate that across the full range of divergence both

195 premating and postzygotic $\mathrm{Rl}$ are stronger between sympatric than allopatric species for

196 three different animal taxa. Because analysis of RI across the full range of divergence

197 may conflate conditions for co-occurrence of reproductively isolated lineages with the

198 conditions that accelerate speciation, we next assessed RI for the most recently

199 diverged species.

200

Comparisons of RI in sympatry depend on divergence time

202 We first restricted our dataset to recently diverged Drosophila pairs as defined by

$203 \mathrm{C} \& \mathrm{O}\left(D_{\mathrm{Nei}}<0.5\right)$ to test if premating, but not postzygotic, $\mathrm{Rl}$ is strengthened at earlier 
204 stages of divergence. Both premating $(W=479.5 ; P<0.0001)$ and postzygotic $(W=$ $205902.5, P=0.039) \mathrm{RI}$ are stronger in sympatry using the C\&O divergence threshold for 206 young species. However, this result is highly contingent on the arbitrary choice of how 207 to define recently diverged species, such that with a slightly lower threshold $\left(D_{\mathrm{Nei}}=\right.$ 208 0.47) the strength of postzygotic RI in sympatry and allopatry does not differ $(W=881.5$, $209 P=0.05$; Figure S4). In contrast, postzygotic RI between toads is always significantly 210 increased in sympatry relative to allopatry (Table S5). Fitting 4PL regressions to the 211 most recently diverged Drosophila $\left(D_{\mathrm{Nei}}<0.5\right)$ and Bufo pairs (NJ distance $<0.05$;

212 Figure S5) indicates that the inflection point of the regression occurs earlier for 213 sympatric than for allopatric Drosophila $\left(c_{\text {sympatric }}=0.366 ; c_{\text {allopatric }}=0.468 ; W=812,777\right.$; $214 P<0.0001)$ and Bufo $\left(c_{\text {sympatric }}=0.029 ; c_{\text {allopatric }}>0.5\right)$ pairs. Similar analyses were not possible for Lepidopterans because the 4PL regressions did not converge when the dataset included only species pairs with Nei's $D<0.5$.

217 In sum, our results indicate that comparisons of premating and postzygotic RI 218 between sympatric Drosophila depend greatly on divergence time; and while comparisons that include only the most recently diverged Drosophila species support

220 the predictions of reinforcement, elevated postzygotic RI between sympatric Drosophila and Bufo at relatively early stages of divergence implies other mechanisms also contribute to these patterns.

Proxies of the cost of hybridization are correlated with the strength of premating and postzygotic RI in Drosophila

We next tested three additional predictions of reinforcement as the primary driver of strengthened premating $\mathrm{RI}$ in sympatry. First, if premating $\mathrm{RI}$ evolves via reinforcement, then the selection pressure to evolve premating $\mathrm{RI}$ might be a function of the cost of hybridization $(40,41)$. It then follows that if the strength of $\mathrm{RI}$ differs between reciprocal crosses, the cross direction with stronger postzygotic $\mathrm{RI}$, and thus a higher cost to hybridization, should also display stronger premating RI (42). A pattern of

232 'concordant asymmetries' (i.e., asymmetries in premating and postzygotic RI in the 233 same direction) has been observed in selected sympatric Drosophila species (16 
234 species pairs), but not in allopatric ones (20 species pairs), which is argued to uniquely support the prevalence of reinforcement ((42) but see (16)). with strong postzygotic $\mathrm{RI}$ should show relatively strong premating $\mathrm{RI}$ ), testing for a positive correlation between the magnitude of premating and postzygotic $\mathrm{Rl}$ asymmetries in Drosophila. Across all of the data, the strength of the asymmetry in postzygotic $\mathrm{RI}$ predicts the strength of the asymmetry in premating $\mathrm{RI}$ in sympatry $(N=$ $\left.73, F_{1,71}=8.037, P=0.006\right)$, but not in allopatry $\left(N=91, F_{1,89}=0.002, P=0.964\right)$, but

242 the proportion of premating RI asymmetry explained by postzygotic $\mathrm{Rl}$ asymmetry in sympatry is low $\left(R^{2}=0.089\right.$, Figure 3A). Figure S6 shows that the association between

244 the strength of premating and postzygotic RI asymmetries is sensitive to inclusion

245 parameters in the study. If only cases where the magnitude of postzygotic RI is asymmetric are included, the association between the magnitude of premating and postzygotic $\mathrm{Rl}$ asymmetries is not significant (sympatry: $F_{1,11}=1.381 ; P=0.265$; allopatry: $F_{1,16}=1.905, P=0.186$; dashed lines in Figures $3 \mathrm{~A}$ ). A partial correlation while controlling for genetic distance shows that the magnitude of premating and postzygotic $\mathrm{Rl}$ asymmetries are only weakly correlated $(\rho=0.192, P=0.050)$. Thus, even if concordant asymmetries are taken as incontrovertible evidence for reinforcement (see below), we interpret these results as supporting that other mechanisms also contribute to strengthened RI between sympatric Drosophila species.

Second, because the risk of hybridization is proportional to the extent of geographic range overlap, in sympatry reinforcement should produce a positive relationship between the strength of premating $\mathrm{RI}$ between species and the proportion of their range overlap. This relationship should not exist for postzygotic $\mathrm{RI}$ since there is no impetus for reinforcement to act on it (1). Consistent with the reinforcement hypothesis and findings of previous reports (43), we find that range overlap is positively associated with premating $\mathrm{RI}$ in Drosophila, even after controlling for genetic distance (Linear Model: $F_{1,255}: 50.809, P=1.044 \times 10^{-11}$; Figure 3B). However, the proportion of

262 geographic range overlap is also positively associated with the magnitude of postzygotic

$263 \mathrm{Rl}$ in Drosophila $\left(F_{1,105}=14.098, P=2.85 \times 10^{-4}\right.$; Figure 3C). Table S6 shows the

264 results for the linear models. Notably, there is no relationship between geographic range 
overlap and the strength of premating or postzygotic $\mathrm{Rl}$ in species whose ranges overlap more than 5\%, suggesting the Drosophila result is driven by species pairs that are mostly allopatric (Table S7). This result suggests that in some instances overlap might drive the evolution of both premating and postzygotic RI, and is thus inconsistent with the predictions of reinforcement being the exclusive driver of premating $\mathrm{Rl}$ in sympatry.

Finally, and as C\&O (1) noted, if reinforcement is responsible for the strengthened $\mathrm{RI}$ in sympatry, then sympatric pairs should display a range of $\mathrm{RI}$ values that is higher and outside the range observed between allopatric pairs because natural selection will lead to RI after secondary contact. Conversely, if differential fusion and/or extinction is responsible for the pattern of stronger $\mathrm{RI}$ in sympatry $(29,30)$, the range of $\mathrm{RI}$ observed among sympatric pairs should be a subset of the RI observed among allopatric pairs because species pairs with weak $\mathrm{RI}$ will go extinct (1). The range of $\mathrm{RI}$ among sympatric pairs is similar to that of allopatric pairs (i.e., both are bounded by 0 and 1), including with the Coyne and Orr (1) threshold for young species (Nei's $D<0.5$; Figure S7). (Please note that the range is not equivalent for Drosophila species pairs younger than Nei's $D<0.25$ ((42); see Discussion). Sympatric and allopatric Bufo pairs show a similar range of postzygotic $\mathrm{RI}$ regardless of divergence, but this is not the case for Lepidopterans (Figure S8). We interpret these results as indicating that multiple mechanisms likely contribute to increased premating $\mathrm{RI}$ in sympatry.

\section{Discussion}

Comparative studies are a powerful tool to study how $\mathrm{Rl}$ accrues, divergence proceeds, and speciation occurs. Groundbreaking analyses by C\&O and others broadly support the hypothesis that reinforcement contributes significantly to Drosophila speciation, but as C\&O (1) noted "enhanced prezygotic RI could result from a process of fusion or extinction in sympatry" because "any factor that reduces gene flow should

293 premating $\mathrm{Rl}$ in sympatry, in the absence of reinforcement (29). We discuss several 294 lines of evidence that indicate multiple mechanisms likely contribute to increased RI in sympatry. 
First, both premating and postzygotic RI are increased in sympatry for recently diverged Drosophila species as defined by Coyne and Orr (1), but at slightly lower divergence thresholds increased postzygotic $\mathrm{RI}$ in sympatry is no longer statistically significant. This pattern generally supports the predictions of reinforcement, while highlighting that comparisons of $\mathrm{RI}$ in sympatry depend greatly on divergence time. Postzygotic $\mathrm{Rl}$ is also increased in sympatry between Lepidopteran and between the most recently diverged Bufo species. (Premating data are not available in either system.) Elevated postzygotic RI in sympatry for relatively recently diverged pairs in three divergent taxa implies multiple mechanisms combine to produce these patterns.

Second, the absence of very recently diverged and strongly isolated allopatric Drosophila species pairs has been argued as support for reinforcement and against fusion and extinction. If differential fusion and extinction contribute to elevated premating $\mathrm{Rl}$ in sympatry, we should also observe strongly prezygotic isolated pairs in allopatry. In contrast to this prediction, Yukilevich ((42), Figure 1) reported a paucity of very recently diverged allopatric Drosophila pairs $(D<0.25)$ with strong premating $\mathrm{Rl}$ (i.e., a "Templeton gap"). While this result supports the predictions of reinforcement, we argue it must depend on species definitions. For example, multiple instances in the literature report strong assortative mating between populations of the same species (i.e., D. lacertosa: Table 2 in (44); D. yakuba: Table 4 in (45); and 14 other species in (46)). Researchers are more likely to classify strongly isolated sympatric groups as different taxonomic species since their isolation has been "tested" in secondary contact. This creates an inherent bias when making these comparisons at the early stages of divergence, producing the illusion of a gap. This bias and the existence of strongly isolated allopatric populations within species that are weakly differentiated leads us to conclude that support for the Templeton gap in Drosophila is less compelling than previously argued. Notably, including these intraspecific observations lowers the inferred $c$ value for allopatric species pairs, but not enough to change the conclusion that premating $\mathrm{Rl}$ accumulates faster in sympatric species (Figure S9). between species pairs have also been proposed as support for reinforcement of 
reciprocal Drosophila crosses that produce strong postzygotic RI also produce strong premating RI; however, asymmetry in postzygotic RI explains only $9 \%$ of the variance in premating $\mathrm{Rl}$ asymmetries. While this pattern might be explained by the predictions of reinforcement, it might also be explained by differential fusion and/or extinction. In instances where premating and postzygotic RI act jointly to prevent fusion (47), one could also expect concordant asymmetries even if reinforcement is not driving the pattern of enhanced RI in sympatry. The same pattern of concordant asymmetries could also be explained by slight asymmetries in gene flow, which could produce asymmetrical accumulation of intrinsic postzygotic RI (16). Given that we also observe a strong effect of geographic range overlap on the evolution of premating $\mathrm{RI}$ and postzygotic $\mathrm{RI}$, these results provide further evidence that no single mechanism explains increased RI in sympatry.

What then explains our observation of stronger postzygotic RI in sympatry for three divergent taxa? One possibility is that postzygotic RI also evolves through pervasive reinforcing selection. This would explain the faster evolution of premating and postzygotic RI in sympatry and the strong effect of geographic overlap in both barriers. Reinforcement of postmating $\mathrm{Rl}$ has been demonstrated in Drosophila $(48,49)$ and Neurospora $(50,51)$, but in all cases the reinforced barriers act prior to zygote formation (i.e., postmating-prezygotic barriers). Even though hybrid inviability has been hypothesized to potentially evolve through selection against hybrids (52), reinforcement should only occur for hybrid inviability if aborting the hybrid embryos might represent an advantage to the parents. (No similar rationale has been proposed for hybrid sterility.) Since Drosophila, Lepidopterans, and toads all show external development, there is no impetus for selection to reinforce postzygotic RI.

A second possibility is that stronger postzygotic $\mathrm{RI}$ in sympatry results in part from fusion and/or extinction $(29,30,53)$. In cases when postzygotic $R I$ is increased in sympatry, and specifically in cases when both premating and postzygotic $\mathrm{RI}$ are stronger in sympatry, differential fusion is a more likely driver of the pattern of enhanced $\mathrm{RI}$ in sympatry than reinforcement (1). This idea has been disfavored because C\&O did not observe elevated postzygotic RI for recently diverged sympatric Drosophila species, and because of the Templeton gap (see above). Our observation of increased 
postzygotic $\mathrm{RI}$ between relatively recently diverged Drosophila, butterflies, and between recently diverged toads implies a role for fusion, extinction, and/or other mechanisms.

A final, and under-discussed possibility, is that the pattern of stronger $\mathrm{Rl}$ in sympatry is due to gene flow which might lead to a systematic underestimation of the age of sympatric species. This will disproportionately affect recently diverged sympatric species as the extent of gene flow seems to be negatively correlated with the divergence age between the parental species $(54,55)$. This possibility might affect our inference of stronger postzygotic $\mathrm{RI}$ in sympatry and specifically create the appearance of stronger RI at low genetic distances. We argue this is an unlikely possibility. In the case of Drosophila, the vast majority of introgression is purged rapidly (i.e., within the first ten generations) after admixture (56-58). The percentage of the genome that shows evidence of gene exchange among Drosophila species pairs ranges between $0.1 \%$ and $5 \%(59-61)$. Nonetheless, the effect of introgression on point measurements of differentiation deserves a systematic treatment.

\section{Conclusions}

Research from the last three decades has revealed dozens of cases of strengthened RI in sympatry resulting from reinforcement (reviewed in (26-28)). Experimental evolution has also demonstrated that $\mathrm{RI}$ can evolve rapidly if selection against hybridization is strong, lending additional support to the possibility of reinforcement in nature $(48,62-65)$. Using an expanded dataset, our Drosophila analyses generally agree with the main finding of C\&O that premating, but not postzygotic, $\mathrm{Rl}$ is increased between the most recently diverged sympatric species pairs. However, the dependence of this result on divergence time, our observation of increased postzygotic $\mathrm{RI}$ between sympatric butterflies and toads, and the other lines of evidence discussed above support that fusion, extinction, and/or other mechanisms must combine to produce these patterns.

Notably, strengthened RI does not seem to be a deterministic outcome of sympatry. Glycine and Silene plant pairs show similar levels of postmating-prezygotic RI in sympatry and allopatry (66). A concerted effort to compare the prevalence of strengthened $\mathrm{Rl}$ in sympatry, and its potential causes across divergent taxa, is sorely 
needed. We expect that those analyses will reveal more support that no single mechanism underlies elevated $\mathrm{RI}$ in sympatry. While comparative studies of $\mathrm{RI}$ are useful for testing hypotheses across diverse groups of organisms, the results of our analyses suggest to us that we need a better understanding of the exact mechanisms underlying the evolution of $\mathrm{RI}$ in sympatry.

\section{MATERIALS AND METHODS}

\section{Datasets}

Our goal was to study whether the rate of evolution of postzygotic $R I$ is similar in sympatric and allopatric animal species pairs. We gathered seven datasets that included metrics of RI (Table S8). Five of these datasets included estimates of genetic distance and the extent of geographic range overlap (i.e., whether species pairs are sympatric or allopatric). Two of these studies have fewer than five species pairs in each category, leaving only three datasets that include systematically gathered metrics of postzygotic RI in sympatric and allopatric species (Table S8). First, we used the data on over 630 Drosophila-species pairs from Yukilevich (42), the most extensive compilation

407 of measurements of reproductive RI in Drosophila. This compendium included all of the 408 data from C\&O (1, 2), data on postzygotic RI across Drosophila from Bock (67), and 409 new data collected by Yukilevich (42). In total, the dataset includes 288 interspecific 410 hybridizations with estimates of genetic distance. Of these, 140 species pairs were 411 classified as sympatric and 148 as allopatric. The second dataset was postzygotic RI in 412 Lepidopterans (data from (68)). The dataset includes 212 interspecific hybridizations, 68 413 of which have measurements of genetic distance. Of these, 52 species pairs were 414 classified as sympatric and 16 as allopatric. The final dataset, was postzygotic RI in 415 toads (data from $(39,69)$ ). This dataset includes 669 interspecific hybridizations, all of 416 which have measurements of genetic distance (calculated from a neighbor-joining tree, 417 (39)). Ninety-five species pairs were classified as sympatric and 574 as allopatric. Only 418 the Drosophila dataset includes estimates of premating RI. Please note that the 419 measurement of genetic distance between toad species (derived from a neighbor- 
420 joining tree) is not equivalent to that in Drosophila and Lepidopterans (Nei's D), so we refrain from doing among comparison groups between insects and anurans.

Model fitting

We investigated the relationship between the strength of $\mathrm{RI}$ (either premating or postzygotic in Drosophila, or postzygotic in Lepidopterans and toads) and the genetic distance between the parental species. First, we compared the fit of three models to the accumulation of RI with genetic distance: linear, logistic increase, and a four-parameter logistic (i.e., dose-response) curve. We fit the linear model using the Im (library 'stats') function in $\mathrm{R}(70)$ and the other two models using the nlsLM (library 'minpack.Im', (71)) function in $\mathrm{R}$. The logistic models followed the form:

$$
\text { Isolation } \sim \frac{1}{1+e^{-((a \times \text { Genetic distance })+b)}}
$$

where Isolation is either a premating or postzygotic RI metric, $a$ is when the genetic distance is zero, and $b$ adjusts how quickly the probability changes with a single-unit change.

The four-parameter logistic models had the form:

$$
\text { Isolation } \sim d+\frac{a-d}{1+\left(\frac{\text { Genetic distance }}{c}\right)^{b}}
$$

443 where $a$ is the magnitude of isolation in a given cross at the minimum genetic distance

444 (here where Nei's $D=0$ ), $b$ is the rate of increase in $\mathrm{RI}$ at point $c$, the inflection point of

445 the decay curve, and $d$ is the maximum $\mathrm{Rl}$ that can be obtained at high genetic

446 distance. This model allows for an initial period where F1 hybrids do not suffer fitness

447 consequences $(34,38)$ and includes that $\mathrm{RI}$ must asymptote at a value no higher than 1

448 (i.e., more genetic changes contributing to isolation become redundant as two lineages

449 cannot be more isolated than completely isolated). Since nonlinear logistic regression 
450 has difficulties optimizing the values for each of the four constants in the equations, we

451 tried 10 starting values per constant and found the model with the lowest Akaike

452 Information Criterion (AIC, (72)) with the function 'AIC', (library 'stats'; (70)).

453 To determine which of the four models best describes the relationship between

454 isolation and genetic divergence we used AICs (72). To find the confidence intervals of

455 the logistic regressions we bootstrapped the datasets 1,000 times using the function

456 nlsBoot (library('nlstools', (73)). Finally, we compared the confidence intervals using

457 Wilcoxon tests of the bootstrap values for each coefficient using the function wilcox.test

458 (library 'stats', (70)).

\section{Phylogenetic independence}

Ideally, our estimates of RI would be phylogenetically independent from all others.

463 However, our Drosophila dataset contains multiple species pairs with phylogenetic

464 relationships that are not evolutionarily, and thus might not be statistically, independent.

465 To account for this lack of independence, we used clade-level and species-level

466 sampling schemes for Drosophila, and a species-level sampling scheme for Bufo frogs

$467(9,66)$. For the species group-level sampling scheme in Drosophila, we sampled a

468 single cross from within each monophyletic clade of Drosophila (affinis, ananassae,

469 athabasca, buzzatii, melanogaster, mesophragmatica, montium, mulleri, nasuta,

470 obscura, planitibia, pseudobscura, repleta, takahashii, virilis, and willistoni; Suvorov et

471 al. 2020) resulting in two datasets: one allopatric and one sympatric, each composed of

472 about 16 species pairs. We subsampled the whole dataset 1,000 times and recalculated

473 the value of $c$, the inflection point, for each iteration as described above (See Model

474 fitting). We recorded the number of iterations that did not converge (i.e., the inferred $c$

475 value was outside of bounds of the range of the function, in this case larger than the

476 maximum Nei's $D$ value), but restricted comparisons between $c_{\text {Sympatric }}$ and $c_{\text {Allopatric }}$ to

477 regressions that converged. We followed a similar approach for Lepidopterans

478 subsampling by genus (Anartia, Anthocharis, Callosamia, Choristoneura, Colias, Erebia,

479 Heliconius, Helicoverpa, Heliothis, Hyalophora, Papilio, Phyciodes, Pieris, Pontia, and

480 Ypomeneuta). We did not use this approach for Bufo toads because a large proportion 
of the hybridizations involved species from different Bufo sensu lato genera (Bufo sensu stricto, Sclerophrys, Schismaderma, Rhinella, Incillius, and Anaryxus). approach proposed by Castillo (39) for the three different taxa. We fitted a generalized linear mixed model using Markov Chain Monte Carlo to study the relationship between $\mathrm{RI}$ and genetic distance for sympatric and allopatric species. We used the function ginv (library MASS, (74)) to find the generalized inverse of the (1-genetic distance) matrix (39). We used the package MCMCgImm (75) and fitted a linear model, in which the magnitude of isolation (premating or postzygotic for Drosophila, and postzygotic for Lepidopterans and Bufo) was the response variable, genetic distance was a predictor variable, geographic overlap (i.e., whether a species pair was sympatric or allopatric) was a fixed effect, and the phylogenetic variance matrix was a random effect. The model also included an interaction between transformed genetic distance and geographic overlap. We ran two independent MCMC chains. To determine if the model converged in each of the two chains, we used the function gelman.diag (library coda, (76)). A chain was considered converged if all scale reduction factors for all variables (both fixed and random effects) were $\leq 1.1$ for each of the two chains. We calculated the 95\% confidence interval for the intercept and the slope using the function HPDinterval (library coda, (76)).

\section{Asymmetries and geographic range}

Asymmetries and overlap of geographic range are two proxies of the risk and cost of hybridization. For sympatric species pairs where postzygotic $\mathrm{RI}$ is stronger for one of the two reciprocal crosses, premating $\mathrm{Rl}$ should also be asymmetric and stronger for the same cross direction if reinforcement has driven the evolution of premating RI. This correlation should not exist for allopatric species pairs (42). For the Drosophila

507 dataset, we calculated the proportion of the variance in asymmetries in premating $\mathrm{Rl}$ explained by asymmetries in postzygotic $\mathrm{Rl}$ as the adjusted $R^{2}$ from a linear regression.

509 We fit linear models with the function Im (library 'stats', (70)) where the magnitude of the 510 asymmetry in premating $\mathrm{RI}$ was the response, and the magnitude of the postzygotic RI 511 was the only continuous effect $(N=167$ species pairs). We also calculated the partial 
512 correlation coefficient between asymmetry in premating and postzygotic RI while

513 controlling for genetic distance using the function pcor.test (ppcor library, (77)) for a

514 dataset that included the magnitude of both asymmetries and genetic distance $(N=106$

515 species pairs).

$516 \quad$ We used a linear model to study the effect of geographic range overlap on

517 patterns of RI. Since we studied two types of RI barriers, we fitted two different linear

518 models. The approach for the two models was identical and the model with the

519 interaction followed the form:

520

$$
\begin{aligned}
& \text { Isolation } \sim \text { Percentage sympatry } y_{i}+\text { Genetic distance } \\
& +(\text { Percentage sympatry } \times \text { Genetic distance })_{i j}+\text { Error }_{i j}
\end{aligned}
$$

522 where Isolation refers to either a premating or postzygotic RI metric. To assess the 523 significance of the interaction, we followed a maximum-likelihood model simplification 524 approach $(78,79)$, in which we compared the model with the interaction to one without 525 the interaction that followed the form:

$$
\text { Isolation } \sim \text { Percentage sympatry } \text { senetic distance }_{j}+\text { Error }_{i j}
$$

To compare the two linear models, we used the function Irtest (library 'Imtest', (80)).

\section{Acknowledgements}

We thank J. M. Coughlan and A. Dagilis for comments and J. M. Good for helpful

534 discussions. Research reported in this publication was supported by the National

535 Institute of General Medical Sciences of the National Institutes of Health (NIH) under

536 Award Number R01GM121750 to DRM and R35GM124701 to BSC. The content is

537 solely the responsibility of the authors and does not necessarily represent the official

538 views of the $\mathrm{NIH}$. 
FIGURES

FIGURE 1. Both premating and postzygotic RI evolve faster in sympatric than in Drosophila species pairs. Semitransparent lines show 1,000 bootstrapped distributions. For both types of barriers, the inflection point $(c)$ is at lower genetic distances for sympatric than for allopatric species pairs (premating: C; postzygotic: D). The vertical 548 lines show the mean value of the bootstrap coefficients. Figure S1 presents the other 549 three parameters for each of the two models.

A. Premating

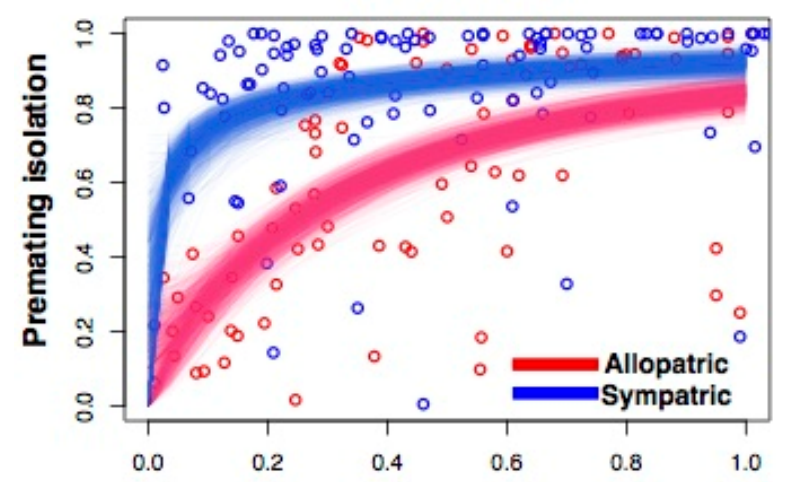

Genetic distance (Nei's D)

\section{Drosophila premating: $c$}

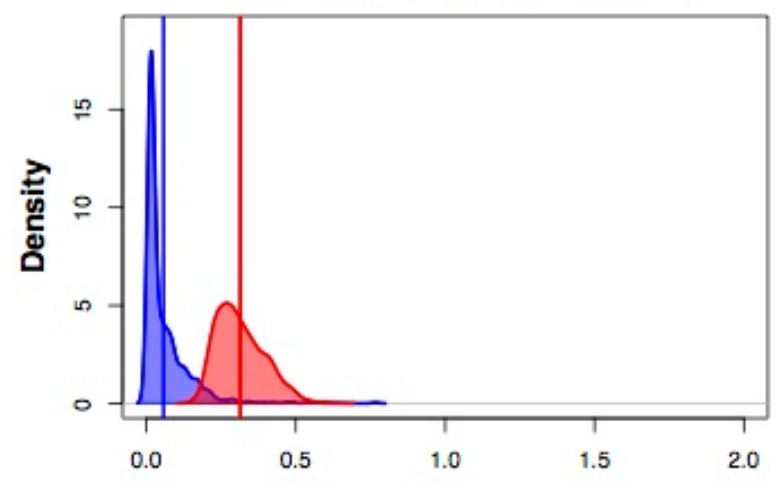

Genetic distance (Nei's D)

\section{B. Postzygotic}

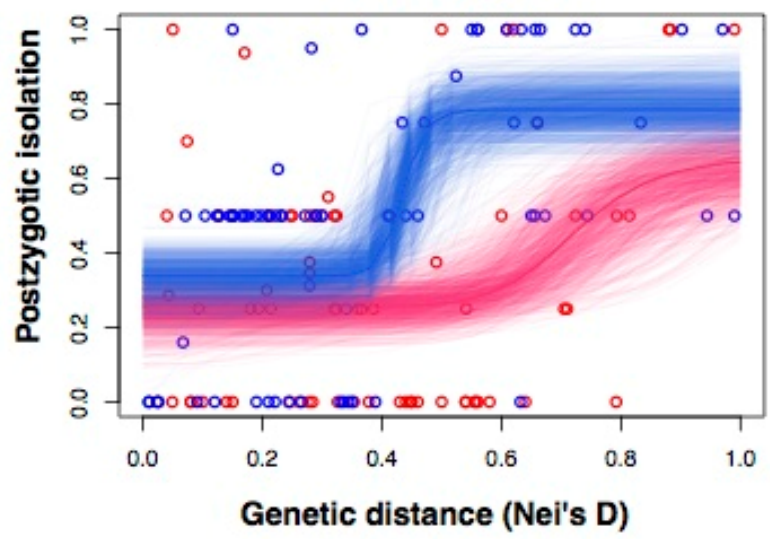

D. Drosophila postzygotic: $c$

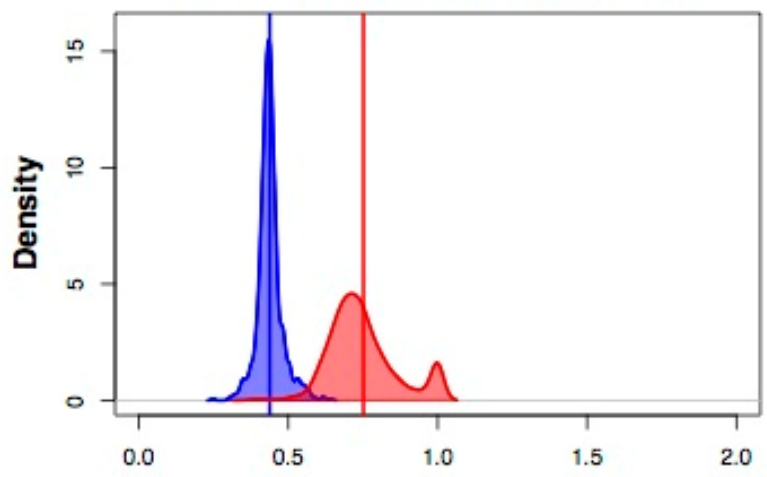

Genetic distance (Nei's D) 


\section{FIGURE 2. Postzygotic RI evolves faster in sympatric than in allopatric}

553 Lepidopteran and Bufo toad species pairs. Four-parameter logistic models for the

554 evolution of postzygotic RI between sympatric (blue) and allopatric (red) Lepidopteran

555 (A) and Bufo (B) species pairs. Semitransparent lines show 1,000 bootstrapped

556 distributions. For both barriers, the inflection point $(c)$ is at lower genetic distances for

557 sympatric than for allopatric species pairs (Lepidopterans: C; Bufo: D). The vertical lines 558 show the mean value of the bootstrap coefficients. Figure S3 presents the other three 559 parameters for each of the two models. Genetic distance is measured as Nei's $D$ for 560 Lepidopterans and from a neighbor-joining tree for Bufo (39).

A. Postzygotic, Lepidopterans

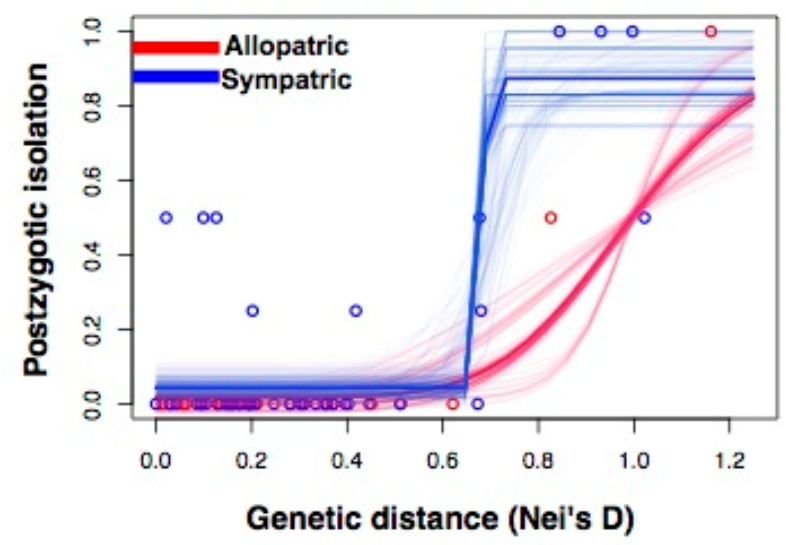

C. Lepidopterans postzygotic: $c$

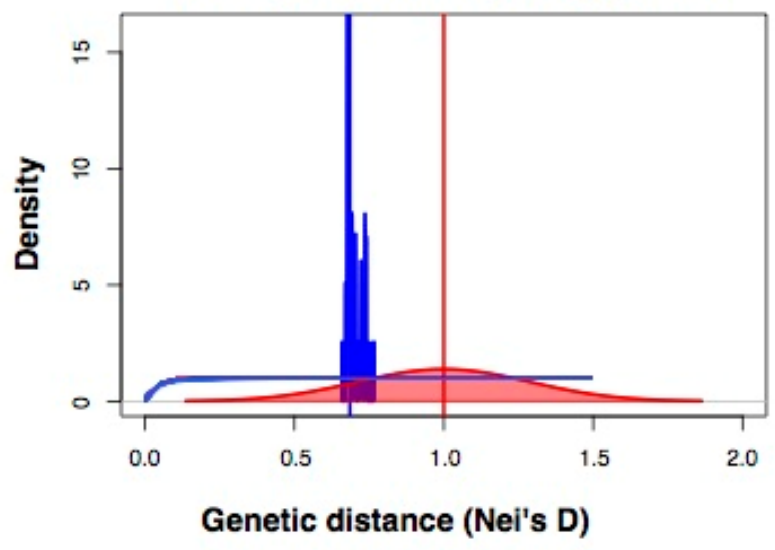

B. Postzygotic, Bufotoads

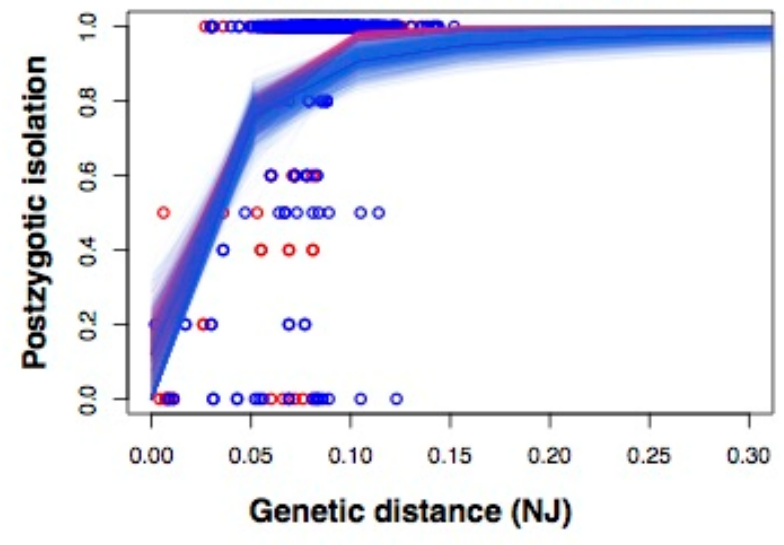

D. Bufo toads postzygotic: $c$

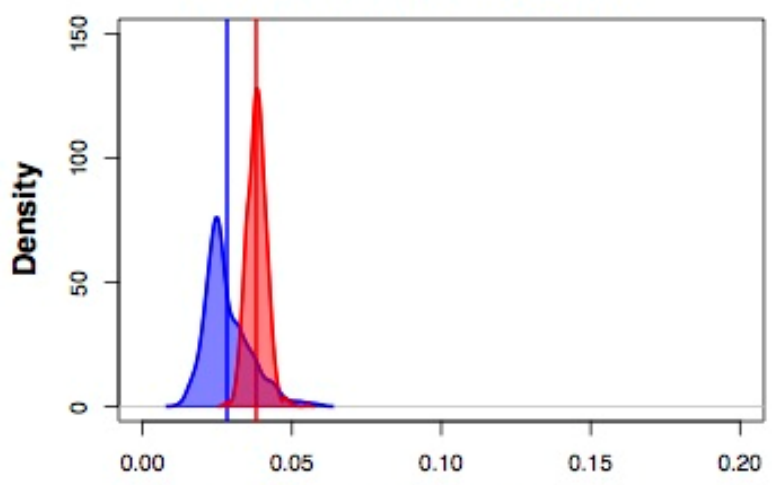

Genetic distance (NJ) 
FIGURE 3. Proxies of the cost of hybridization are correlated with the strength of RI in Drosophila. A. The strength of asymmetries in premating $\mathrm{Rl}$ is weakly correlated with the strength of asymmetries in postzygotic RI in sympatry (solid blue), but not in allopatry (solid red). When completely symmetric pairs (i.e., those where the difference in the strength of RI between reciprocal crosses equals 0 ) are excluded, asymmetries in premating and postzygotic $\mathrm{RI}$ in sympatry are no longer significantly correlated (dashed blue line). Range overlap is correlated with the strength of both premating (B) and postzygotic $\mathrm{RI}(\mathbf{C})$.

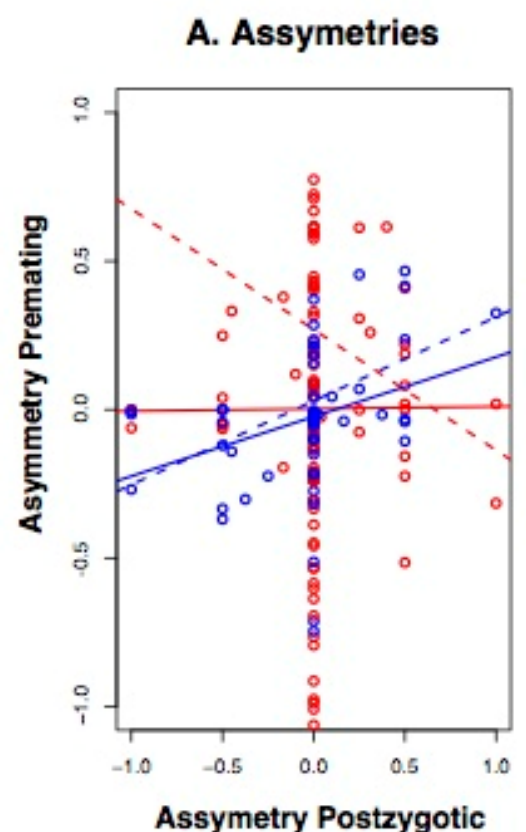

Assymetry Postzygotic
B. Premating vs.geographic range overlap

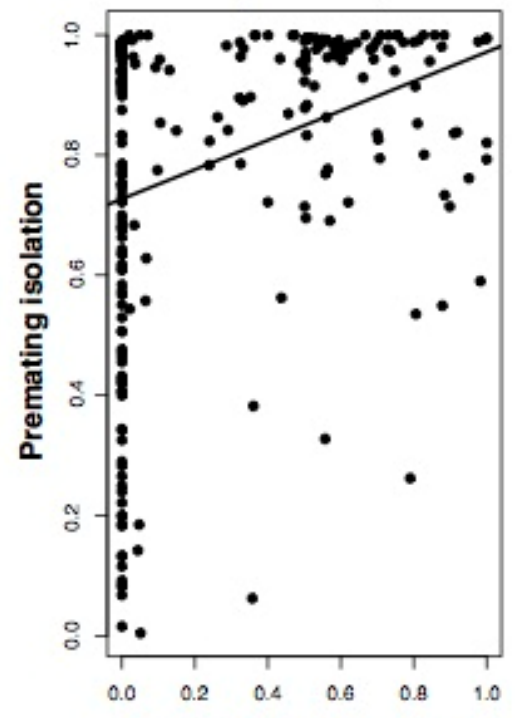

Geographic range overlap
C. Postzygotic vs. geographic range overlap

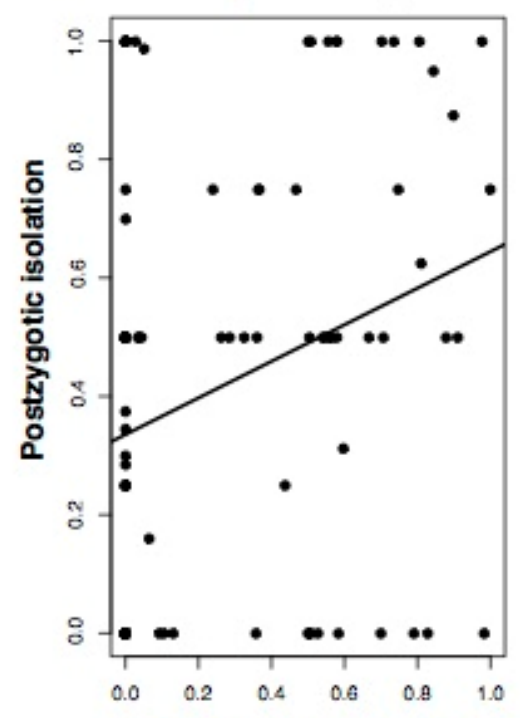

Geographic range overlap 


\section{REFERENCES}

576 1. J. A. Coyne, H. A. Orr, Patterns of Speciation in Drosophila. Evolution 43, 362-381 (1989).

577 2. J. A. Coyne, H. A. Orr, "Patterns of Speciation in Drosophila" Revisited. Evolution 51, 295 $578 \quad$ (1997).

579 3. J. A. Coyne, H. A. Orr, Speciation, Sinauer Associates, Sunderland, MA (2004).

580 4. P. Nosil, Ecological Speciation, Oxford Series in Ecology and Evolution. Oxford University $581 \quad$ Press, New York, NY (2012)

582 5. T. Dobzhansky, Genetics and the Origin of Species (Columbia University Press, 1937).

6. J. M. Sobel, G. F. Chen, L. R. Watt, D. W. Schemske, The biology of speciation. Evolution 64, 295-315 (2010).

7. H. A. Orr, The genetic basis of reproductive isolation: Insights from Drosophila. Proceedings of the National Academy of Sciences 102, 6522-6526 (2005).

8. H. A. Orr, D. C. Presgraves, Speciation by postzygotic isolation: Forces, genes and molecules. BioEssays 22, 1085-1094 (2000).

9. D. A. Turissini, A. A. Comeault, G. Liu, Y. C. G. Lee, D. R. Matute, The ability of Drosophila hybrids to locate food declines with parental divergence. Evolution 71, 960-973 (2017).

10. M. A. McQuillan, T. C. Roth, A. V. Huynh, A. M. Rice, Hybrid chickadees are deficient in learning and memory. Evolution 72:1155-1164 (2018).

11. K. E. Delmore, D. E. Irwin, Hybrid songbirds employ intermediate routes in a migratory divide. Ecology Letters 17, 1211-1218 (2014).

12. D. R. Matute, J. A. Coyne, Intrinsic reproductive isolation between two sister species of Drosophila. Evolution 64, 903-920 (2010).

13. R. S. Burton, Hybrid breakdown in developmental time in the copepod Tigriopus californicus. Evolution 44, 1814-1822 (1990)

14. A. D. Cutter, J. D. Bundus, Speciation and the developmental alarm clock. bioRxiv, Elife, 9, p.e56276 (2020).

15. D. R. Matute, B. S. Cooper, Comparative studies on speciation: 30 years since Coyne and

16. M. Turelli, J. R. Lipkowitz, Y. Brandvain, On the Coyne and Orr-lgin of Species: Effects of intrinsic postzygotic isolation, ecological differentiation, $X$ chromosome size, and sympatry on Drosophila speciation. Evolution 68, 1176-1187 (2014). 
608

609

610

611

612

613

614

615

616

617

618

619

620

621

622

623

624

625

626

627

628

629

630

631

632

633

634

635

636

637

638

639

640

641

642

18. T. G. Lima, Higher levels of sex chromosome heteromorphism are associated with markedly stronger reproductive isolation. Nature Communications 5, 4743 (2014).

19. B. M. Fitzpatrick, Molecular correlates of reproductive isolation. Evolution 56, 191-198 (2002).

20. B. M. Fitzpatrick, M. Turelli, The geography of mammalian speciation: Mixed signals from phylogenies and range maps. Evolution 60, 601-615 (2006).

21. D. J. Funk, P. Nosil, W. J. Etges, Ecological divergence exhibits consistently positive associations with reproductive isolation across disparate taxa. Proc. Natl. Acad. Sci. U.S.A. 103, 3209-3213 (2006).

22. D. L. Rabosky, D. R. Matute, Macroevolutionary speciation rates are decoupled from the evolution of intrinsic reproductive isolation in Drosophila and birds. PNAS 110, 1535415359 (2013).

23. S. Gourbière, J. Mallet, Are species real? The shape of the species boundary with exponential failure, reinforcement, and the "missing snowball." Evolution 64, 1-24 (2010).

24. J. M. Coughlan, D. R. Matute, The importance of intrinsic postzygotic barriers throughout the speciation process. Philosophical transactions of the Royal Society of London. Series B, Biological sciences 375: 20190533 (2020) https:/doi.org/10.1098/rstb.2019.0533.

25. M. A. Noor, Reinforcement in speciation. Trends Ecol. Evol. (Amst.) 10, 492 (1995).

26. M. R. Servedio, M. A. F. Noor, The role of reinforcement in speciation: Theory and data. Annual Review of Ecology, Evolution, and Systematics 34, 339-364 (2003).

27. R. Hopkins, Reinforcement in plants. New Phytol. 197: 1095-1103 (2013).

28. E. J. Hudson, T. D. Price, Pervasive reinforcement and the role of sexual selection in biological speciation Journal of Heredity, 105: 821-833 (2014).

29. A. R. Templeton, Mechanisms of Speciation - A Population Genetic Approach. Annual Review of Ecology and Systematics 12, 23-48 (1981).

30. R. Butlin, A new approach to sympatric speciation. Trends Ecol. Evol. (Amst.) 2, 310-311 (1987).

31. T. C. Mendelson, B. D. Inouye, M. D. Rausher, Quantifying patterns in the evolution of reproductive isolation. Evolution 58, 1424-1433 (2004).

32. D. I. Bolnick, T. J. Near, Tempo of hybrid inviability in Centrarchid fishes (Teleostiei: Centrarchidae). Evolution 59, 1754 (2005).

33. A. C. R. Lackey, J. W. Boughman, Evolution of reproductive isolation in stickleback fish. Evolution 71, 357-372 (2017).

34. A. J. Dagilis, M. Kirkpatrick, D. I. Bolnick, The evolution of hybrid fitness during speciation. PLOS Genetics 15, e1008125 (2019). 
643

644

645

646

647

648

649

650

651

652

653

654

655

656

657

658

659

660

661

662

663

664

665

666

667

668

669

670

671

672

673

674

675

676

35. S. Edmands, Does parental divergence predict reproductive compatibility? Trends in Ecology \& Evolution 17, 520-527 (2002).

36. H. A. Orr, The population genetics of speciation: the evolution of hybrid incompatibilities. Genetics 139, 1805-1813 (1995).

37. H. A. Orr, L. H. Orr, Waiting for speciation: The effect of population subdivision on the time to speciation. Evolution 50, 1742-1749 (1996).

38. H. A. Orr, M. Turelli, The evolution of postzygotic isolation: accumulating DobzhanskyMuller incompatibilities. Evolution 55, 1085-1094 (2001).

39. D. M. Castillo, Factors contributing to the accumulation of reproductive isolation: A mixed model approach. Ecology and Evolution 7, 5808-5820 (2017).

40. S. S. Suni, R. Hopkins, The relationship between postmating reproductive isolation and reinforcement in Phlox. Evolution 72, 1387-1398 (2018) https:/doi.org/10.1111/evo.13507.

41. N. Poikela, J. Kinnunen, M. Wurdack, H. Kauranen, T. Schmitt, M. Kankare, R.R. Snook, and A. Hoikkala. Strength of sexual and postmating prezygotic barriers varies between sympatric populations with different histories and species abundances. Evolution 73, 1182-1199 (2019).

42. R. Yukilevich, Asymmetrical patterns of speciation uniquely support reinforcement in Drosophila. Evolution 66, 1430-1446 (2012).

43. P. Nosil, Degree of sympatry affects reinforcement in Drosophila. Evolution 67, 868-872 (2013).

44. L. P. He, H. A. Watabe, Y. P. Zhang, T. Aotsuka, Karyotype differentiation and reproductive isolation among natural populations of Drosophila lacertosa. Cell research 13, 491-497 (2003).

45. B. Denis, A. L. Rouzic, C. Wicker-Thomas, Hydrocarbon patterns and mating behaviour in populations of Drosophila yakuba. Insects 6, 897-911 (2015).

46. I. Marin, Sexual isolation in Drosophila I. Theoretical models for multiple-choice experiments. Journal of theoretical biology 152, 271-284 (1991).

47. M. R. Servedio, G.-P. Saetre, Speciation as a positive feedback loop between postzygotic and prezygotic barriers to gene flow. Proc. Biol. Sci. 270, 1473-1479 (2003).

48. D. R. Matute, Reinforcement of gametic isolation in Drosophila. PLOS Biology 8, e1000341 (2010).

49. D. M. Castillo, L. C. Moyle, Conspecific sperm precedence is reinforced, but postcopulatory sexual selection weakened, in sympatric populations of Drosophila. Proceedings of the Royal Society B: Biological Sciences 286, 20182535 (2019). 
677

678

679

680

681

682

683

684

685

686

687

688

689

690

691

692

693

694

695

696

697

698

699

700

701

702

703

704

705

706

707

708

709

710

711

712

50. E. Turner, D. J. Jacobson, J. W. Taylor, Reinforced postmating reproductive isolation barriers in Neurospora, an Ascomycete microfungus. Journal of Evolutionary Biology 23, 1642-1656 (2010).

51. E. Turner, D. J. Jacobson, J. W. Taylor, Genetic Architecture of a reinforced, postmating, reproductive isolation barrier between Neurospora species indicates evolution via natural selection. PLOS Genetics 7, e1002204 (2011).

52. J. A. Coyne, The evolutionary origin of hybrid inviability. Evolution 28, 505-506 (1974).

53. J. M. Rhymer, D. Simberloff, Extinction by hybridization and introgression. Annual Review of Ecology and Systematics 27, 83-109. (1996)

54. M. R. Kronforst, M.E. Hansen, N.G. Crawford, J.R. Gallant, W. Zhang, R.J. Kulathinal, D.D. Kapan, and S.P. Mullen. Hybridization reveals the evolving genomic architecture of speciation. Cell Reports 5, 666-677 (2013).

55. J. A. P. Hamlin, M. S. Hibbins, L. C. Moyle, Assessing biological factors affecting postspeciation introgression. Evolution Letters 4, 137-154 (2020).

56. C. Veller, N. B. Edelman, P. Muralidhar, M. A. Nowak, Recombination, variance in genetic relatedness, and selection against introgressed DNA. bioRxiv, 846147 (2019).

57. D. R. Matute, A.A. Comeault, E. Earley, A. Serrato-Capuchina, D. Peede, A. MonroyEklund, W. Huang, C.D. Jones, T.F. Mackay, and J.A. Coyne. Rapid and predictable evolution of admixed populations between two Drosophila species pairs. Genetics 214, pp.211-230 (2020)

58. A. Suvorov, B.Y. Kim, J. Wang, E.E. Armstrong, D. Peede, E. R. D'Agostino, D.K. Price, P. Wadell, M. Lang, V. Courtier-Orgogozo, J.R. David, Petrov, D., Matute D.R., Schrider D.R. and Comeault A.A. Widespread introgression across a phylogeny of 155 Drosophila genomes. bioRxiv, 2020.12.14.422758 (2020).

59. R. J. Kulathinal, L. S. Stevison, M. A. F. Noor, The genomics of speciation in Drosophila: Diversity, divergence, and introgression estimated using low-coverage genome sequencing. PLoS Genetics 5 (2009).

60. D. Garrigan, S.B. Kingan, A.J. Geneva, P. Andolfatto, A.G. Clark, K.R. Thornton, and D.C. Presgraves, Genome sequencing reveals complex speciation in the Drosophila simulans clade. Genome Research 22, 1499-1511 (2012).

61. D. A. Turissini, D. R. Matute, Fine scale mapping of genomic introgressions within the Drosophila yakuba clade. PLOS Genetics 13, e1006971 (2017).

62. K. F. Koopman, natural selection for reproductive isolation between Drosophila pseudoobscura and Drosophila persimilis. Evolution 4, 135-148 (1950).

63. A. Fukatami, D. Moriwaki, Selection for sexual isolation in Drosophila melanogaster by a modification of Koopman's method. 遺伝學雑誌 45, 193-204 (1970). 
64. M. Higgie, S. Chenoweth, M. W. Blows, Natural Selection and the reinforcement of mate recognition. Science 290, 519-521 (2000).

65. D. R. Matute, Reinforcement can overcome gene flow during speciation in Drosophila. Current Biology 20, 2229-2233 (2010).

66. L. C. Moyle, M. S. Olson, P. Tiffin, Patterns of reproductive isolation in three angiosperm genera. Evolution (2004) 58, 1195-1208.

67. I. R. Bock, "Interspecific Hybridization in the Genus Drosophila" in Evolutionary Biology: Volume 18, M. K. Hecht, B. Wallace, G. T. Prance, Eds. (Springer US, 1984), pp. 41-70.

68. D. C. Presgraves, Patterns of Postzygotic Isolation in Lepidoptera. Evolution 56, 11681183 (2002).

69. J. H. Malone, B. E. Fontenot, Patterns of reproductive isolation in toads. PLOS ONE 3, e3900 (2008).

70. R Core Team, R Development Core Team (2016).

71. T. V. Elzhov, K. M. Mullen, A.-N. Spiess, B. Bolker, minpack.Im: R Interface to the Levenberg-Marquardt Nonlinear Least-Squares Algorithm Found in MINPACK, Plus Support for Bounds (2016) (March 20, 2020).

72. H. Akaike, A new look at the statistical model identification. IEEE Transactions on Automatic Control 19, 716-723 (1974).

73. F. Baty, C. Ritz, S. Charles, M. Brutsche, J.P. Flandrois, and M.L. Delignette-Muller. A Toolbox for Nonlinear Regression in $R \square$ : The Package nlstools. J. Stat. Soft. 66 (2015).

74. W. N. Venables, Modern applied statistics with S / W.N. Venables, B.D. Ripley (2002).

75. J. D. Hadfield, MCMC Methods for Multi-Response Generalized linear mixed models: The MCMCgImm R Package. J. Stat. Soft. 33 (2010).

76. M. Plummer, N. Best, K. Cowles, K. Vines, CODA: convergence diagnosis and output analysis for MCMC. R News 6, 7-11 (2006).

77. S. Kim, ppcor: Partial and Semi-Partial (Part) Correlation (2015) (December 9, 2020).

78. M. J. Crawley, GLIM for Ecologists (1993).

79. M. J. Crawley, Statistical computing: An introduction to data analysis using S-Plus John Wiley \& Sons, Hoboken, NJ (2002).

80. A. T. Hothorn, A. Zeileis, G. Millo, D. Mitchell, Package 'Imtest'. R News (2011). 